\title{
Metallicity and age gradients in round elliptical galaxies ${ }^{\star}$
}

\author{
M. Baes ${ }^{1}$, O. K. Sil'chenko ${ }^{2,3}$, A. V. Moiseev ${ }^{4}$, and E. A. Manakova ${ }^{2}$ \\ 1 Sterrenkundig Observatorium, Universiteit Gent, Krijgslaan 281 S9, 9000 Gent, Belgium \\ 2 Sternberg Astronomical Institute, University av. 13, Moscow 119992, Russia \\ e-mail: olga@sai.msu.su \\ 3 Isaac Newton Institute of Chile, Moscow Branch \\ 4 Special Astrophysical Observatory, Nizhnij Arkhyz 369167, Russia
}

Received 16 November 2006 / Accepted 26 January 2007

ABSTRACT

\begin{abstract}
Aims. We probe the stellar population age and metallicity distributions in nearby elliptical galaxies over the largest extension to date. Methods. Long-slit spectroscopy is made by using the spectrograph SCORPIO of the 6-m telescope of the Special Astrophysical Observatory of the Russian Academy of Sciencies. The Lick indices $\mathrm{H} \beta, \mathrm{Mg}$ b, Fe 5270, and Fe 5335 are calculated along the slit up to radii of 1.3 to $3 r_{\mathrm{e}}$ in 4 galaxies and up to $0.5 r_{\mathrm{e}}$ in the fifth one. The comparison with evolutionary synthesis models of simple stellar populations allows us to disentangle age and metallicity and to measure both.

Results. We have found that the mean stellar age is constant along the radius only in one galaxy out of 5 . The other 4 galaxies demonstrate quite different behaviour of the mean stellar age: the outer parts are older than the centres in 3 cases and younger - in one case. The metallicity gradients cannot be approximated by a single power law over the full radial extension in 4 galaxies of 5 . The inner metallicity gradients within $0.5 r_{\mathrm{e}}$ are all rather steep, steeper than -0.4 metallicity dex per radius dex, and are inconsistent with the origin of the elliptical galaxies by a major merger.
\end{abstract}

Key words. galaxies: elliptical and lenticular, cD - galaxies: evolution

\section{Introduction}

Elliptical galaxies were described by Hubble (1926) as smooth homogeneous stellar systems. However even early photometric studies revealed the existence of colour gradients along the radius in bright ellipticals (Tifft 1969; Burkhead \& Kalinowski 1974), which had been treated later as metallicity gradients because of consistency between the metallicity slopes determined from different-band colour gradients (Sandage \& Visvanathan 1978; Peletier \& Valentijn 1989). But there were also some hints, after comparing colour gradients to absorption-line strength gradients, that there may be also age variations along the radius that make a minor contribution to the colour gradients (Gorgas et al. 1990).

The presence of metallicity gradients along the radius in elliptical galaxies is a crucial test for all models of galaxy formation. The model of monolithic dissipative collapse of a protogalactic gaseous cloud by Larson (1974) predicted the metallicity gradients with the correct sign: during the violent star formation phase over the whole galaxy, the gas enriched by fresh heavy elements fell into the centre and became the material for subsequent star formation. The more elaborated models of dissipative collapse by Carlberg (1984) gave the characteristic value for the final stellar metallicity gradient in the largest elliptical galaxies of -0.5 dex per radius dex, and the dependence of the gradient slope on the galactic mass. Small elliptical galaxies had no metallicity gradient in the models of Carlberg (1984), due to the early occurrence of a galactic wind that stopped the galaxy

* Based on observations collected with the $6 \mathrm{~m}$ telescope of the Special Astrophysical Observatory (SAO) of the Russian Academy of Sciences (RAS) which is operated under the financial support of Science Department of Russia (registration number 01-43). formation. The other main scenario in which elliptical galaxies are formed is the framework of hierarchical merging. In simulations of elliptical galaxies forming by major merging, metallicity gradients are also predicted. In general, the gradients in these simulations are shallower than those formed in the monolithic collapse scenario (White 1980; Barnes 1996; Bekki \& Shioya 1999).

Recently Kobayashi (2004) has considered the evolution of metallicity gradients in the framework of a modern LCDM model of elliptical galaxy formation. The processes considered include monolithic collapse at high redshift and subsequent minor and major mergers followed in some cases by induced starformation bursts. Monte Carlo simulations have given 124 various histories of elliptical galaxy evolution and reproduced the observed variety of metallicity gradients. The extreme case of a galaxy formed by the pure monolithic collapse and then evolving quietly corresponds to a very steep metallicity gradient, about $\Delta[\mathrm{Fe} / \mathrm{H}] / \Delta \log r \approx-1$. During the evolution, the metallicity gradients become shallower when galaxies merge; no galaxy having experienced some major merger has the metallicity gradient steeper than -0.35 dex per radius dex. Among the galaxy ensemble simulated by Kobayashi (2004) the average metallicity gradient is $\Delta[\mathrm{Fe} / \mathrm{H}] / \Delta \log r \approx-0.3$, with a scatter of $\pm 0.2 \mathrm{dex}$ - quite consistent with the observations of colour gradients in elliptical galaxies; no correlation between gradient and galaxy mass is found. These simulations demonstrate that the knowledge of exact values of metallicity gradients in elliptical galaxies is important for probing their evolution. In particular, Kobayashi (2004) suggests that the metallicity gradient in any individual elliptical galaxy is an indicator of its history and may reveal if major mergers were significant during its evolution. 
Table 1. Global parameters of the galaxies.

\begin{tabular}{lllccccc}
\hline \hline & Unit & Source & NGC 2634 & NGC 2810 & NGC 3348 & NGC 4339 & IC 2179 \\
\hline Type & & NED $^{1}$ & E1: & E & E0 & E0 & E1 \\
$V_{r}$ & $\mathrm{~km} \mathrm{~s}^{-1}$ & NED & 2258 & 3572 & 2837 & 1289 & 4336 \\
Distance & $\mathrm{Mpc}$ & LEDA $^{2}$ & 34.7 & 53.5 & 44.3 & $18.2($ Virgo) & 64.6 \\
$D_{25}$ & $\mathrm{arcmin}$ & LEDA & 1.7 & 1.7 & 2.0 & 2.4 & 1.1 \\
$D_{25}$ & $\mathrm{kpc}$ & LEDA & 17.2 & 26.5 & 25.8 & 12.7 & 20.7 \\
$B_{T}^{0}$ & $\mathrm{mag}$ & RC3 & 12.71 & 13.07 & 11.84 & 12.31 & 13.21 \\
$M_{B}$ & $\mathrm{mag}$ & LEDA & -20.0 & -20.7 & -21.7 & -19.3 & -20.9 \\
$(B-V)_{T}^{0}$ & $\mathrm{mag}$ & RC3 & 0.90 & 0.95 & 0.95 & 0.90 & 0.93 \\
$(U-B)_{T}^{0}$ & $\mathrm{mag}$ & RC3 & 0.48 & - & 0.44 & 0.50 & 0.52 \\
$\sigma_{0}$ & $\mathrm{~km} \mathrm{~s}{ }^{-1}$ & LEDA & $182 \pm 4$ & $223 \pm 8$ & $238 \pm 10$ & $113 \pm 4$ & $146 \pm 52$ \\
$r_{\mathrm{e}}$ & arcsec & (see notes) & $18^{4}$ & $14^{5}$ & $22^{4}$ & $26^{5}$ & $15^{5}$ \\
Environment & & NOG $^{6}$ & group, & field? & group, & Virgo & group, \\
& & & off-center & & centre & cluster & near centre \\
\hline
\end{tabular}

${ }^{1}$ NASA/IPAC Extragalactic Database. ${ }^{2}$ Lyon-Meudon Extragalactic Database. ${ }^{3}$ Third Reference Catalogue of Bright Galaxies. ${ }^{4}$ Trujillo et al. (2004). ${ }^{5}$ This work. ${ }^{6}$ Giuricin et al. (2000).

The current observational data provide mean metallicity gradients $\Delta[\mathrm{Fe} / \mathrm{H}] / \Delta \log r$ typically in the range -0.2 to -0.3 (see e.g. Carollo, et al. 1993; Davies, et al. 1993; Kobayashi \& Arimoto 1999; Mehlert et al. 2003), while observational evidence of the predicted correlation between metallicity gradients and galaxy mass or luminosity remains somewhat controversial (Vader et al. 1988; Peletier et al. 1990; Forbes et al. 2005).

Unfortunately, investigating the metallicity gradients in elliptical galaxies is not straightforward, and these observational results should be interpreted with caution. Indeed, most estimates are made under the a priori assumption that the whole colour or absorption-line gradient results from metallicity gradient. For example, we could refer to the paper by Kobayashi \& Arimoto (1999) who summarise a totality of data on metalline index gradients in 80 elliptical galaxies over 20 independent studies and calculate metallicity gradients by using evolutionary synthesis of simple stellar populations (SSP) and by assuming that the stellar populations at any radius in all 80 galaxies under consideration are $17 \mathrm{Gyr}$ old and no age gradient exists. But we know that age-metallicity degeneracy concerns all the optical colours, as well as all the metal-line indices. If one diminishes either metallicity under a constant age or age under a constant metallicity, all the colours would be bluer and all the metal lines would be weaker. So ages and metallicity must be determined simultaneously by confronting for examples metal-line indices to the Balmer lines or to the broad-band optical colours, to make definite conclusions about the metallicity (and age) gradients. Secondly, due to the different scales one must be careful when comparing observationally-determined metallicity gradients to the results of simulation. For example, the metallicity gradients presented by Kobayashi (2004) cover the region out to $2 r_{\mathrm{e}}$, whereas most observational metallicity gradients are derived over a relatively compact region, typically within 0.5 to $1 r_{\mathrm{e}}$. Simple extrapolations of the measured gradients to larger radii are very dangerous possibly leading to erroneous conclusions.

This work presents a precise determination of metallicity and age gradients in five elliptical galaxies made by using Lick-index measurements and by breaking the age-metallicity degeneracy with the two-index diagrams based on the evolutionary synthesis of old SSPs. The other feature of this work is a significant extension of the index profiles: for 4 of 5 galaxies we reach 1.3 to 3 effective radii, whereas most works up to now have considered index gradients within one effective radius. In Sect. 2 we
Table 2. Long-slit spectroscopy of the galaxies.

\begin{tabular}{llccc}
\hline \hline Date & Galaxy & $\begin{array}{c}\text { Exposure } \\
(\mathrm{min})\end{array}$ & $\begin{array}{c}\text { PA (slit) } \\
(\mathrm{deg})\end{array}$ & $\begin{array}{c}\text { Seeing } \\
(\operatorname{arcsec})\end{array}$ \\
\hline 6 Mar. 05 & NGC 3348 & 100 & 34 & 3 \\
7 Mar. 05 & IC 2179 & 220 & 38 & 3 \\
7 Mar. 05 & NGC 2810 & 120 & 63 & 3 \\
20 Apr. 06 & NGC 3348 & 120 & 293 & 2 \\
21 Apr. 06 & NGC 4339 & 40 & 241 & 2 \\
26 Apr. 06 & NGC 2634 & 280 & 45 & 4 \\
\hline
\end{tabular}

present our sample and in Sect. 3 we discuss the observations, data reduction, and the analysis of the spectra. In Sect. 4 we discuss the raw line-index profiles for the galaxies in our sample and convert these indices to age and metallicity gradients using SSP diagrams. These results are discussed in Sect. 5. Section 6 offers a summary.

\section{Sample}

This work is part of the larger project involving complex photometric and spectral studies of nearby round elliptical galaxies. The ultimate aim is to probe the dynamical and stellar structures of elliptical galaxies up to a few effective radii from the centre. Round ellipticals were selected because of their probably spherical shape so the simplest one. For this reason we suggest that one longslit cross-section with an arbitrary orientation of the slit would be enough to obtain a complete picture of radial variations for all kinematical and stellar population properties. The database HYPERLEDA has been used to select all E0 galaxies with a visible ellipticity less than 0.075 (at the 25 th blue isophote) and with the diameters, also at the 25th blue isophote, between 1 and 3 arcmin. The latter condition is imposed to provide sufficient spatial resolution, on one hand, and to match the field of view of the spectrograph SCORPIO, on the other. We must have blank sky measurements for the external parts of the slit to subtract the sky contribution properly. The northern part of the sample lists 18 galaxies. Now we present the first part of the results of the spectral observations for 5 of them. The global characteristics of the considered galaxies are given in Table 1. 

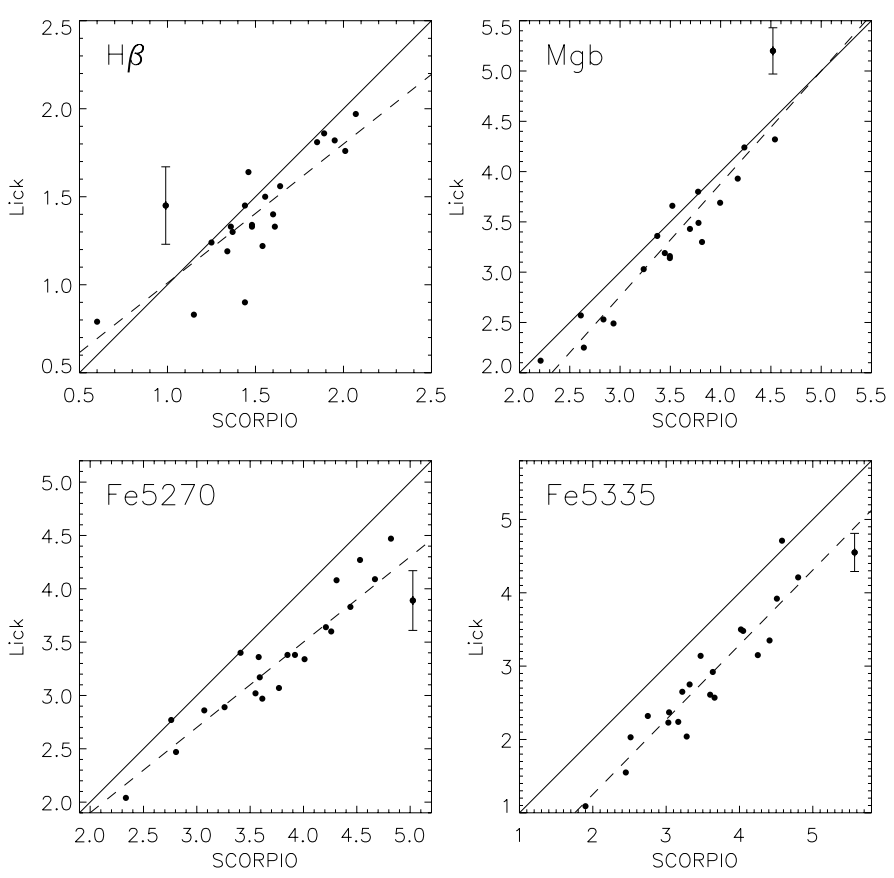

Fig. 1. The correlation of the instrumental absorption-line indices obtained with the spectrograph SCORPIO and the standard Lick indices for 22 stars from the list of Worthey et al. (1994). The thin straight lines are bisectrices of the quadrants, and the dashed lines linear regressions fitted to the dependencies. The mean index error for the measurements of the stars by Worthey et al. (1994) are indicated for a single star in each plot; our errors are lower by one order.

\section{Observations and data reduction}

Long-slit spectral observations were made with the multimode focal reducer SCORPIO (Afanasiev \& Moiseev 2005) installed at the prime focus of the BTA 6-m telescope at the Special Astrophysical Observatory. A description of the SCORPIO instrument can be found at http://www . sao.ru/hq/moisav/scorpio/scorpio.html. For our observations with the SCORPIO we used the phasevolume holographic grating (the grism) VPHG2300 providing the narrow spectral range of 4800-5540 $\AA$; this wavelength region is rich in stellar absorption lines and contains the Lick indices $\mathrm{H} \beta, \mathrm{Mg} b, \mathrm{Fe} 5270$, and Fe 5335 suitable for studying stellar populations. Moreover, the emission lines [OIII] $\lambda \lambda 4959$, 5007 appeared to be strong in some cases; they are used for the gas kinematics study and for estimating the emission contamination level for the Lick index $\mathrm{H} \beta$. The slit width was one arcsec, and the spectral resolution about $2.2 \AA$. The CCD $2 k \times 2 k$ was used as a detector, and the scale across the slit was $0.35^{\prime \prime}$ per pixel. The full log of observations is given in the Table 2. Besides the galaxies, several G- and K-giant stars from the list of Worthey (1994) were observed in the same mode, and their spectra were used for the Lick system calibration and for cross-correlation with the spectra of the galaxies. The preliminary data reduction was performed by means of IDL-based software (Afanasiev \& Moiseev 2005).

During the further reduction we applied logarithmic binning along the slit to provide a sufficient signal-to-noise ratio $(S / N>$ 25-30 per bin). For every galaxy we calculated radial dependencies of the Lick indices $\mathrm{H} \beta, \mathrm{Mg} b, \mathrm{Fe} 5270$, and $\mathrm{Fe} 5335$ that are suitable to determine metallicity, age, and $\mathrm{Mg} / \mathrm{Fe}$ ratio of old stellar populations (Worthey et al. 1994). To calibrate the SCORPIO index system onto the standard Lick one,
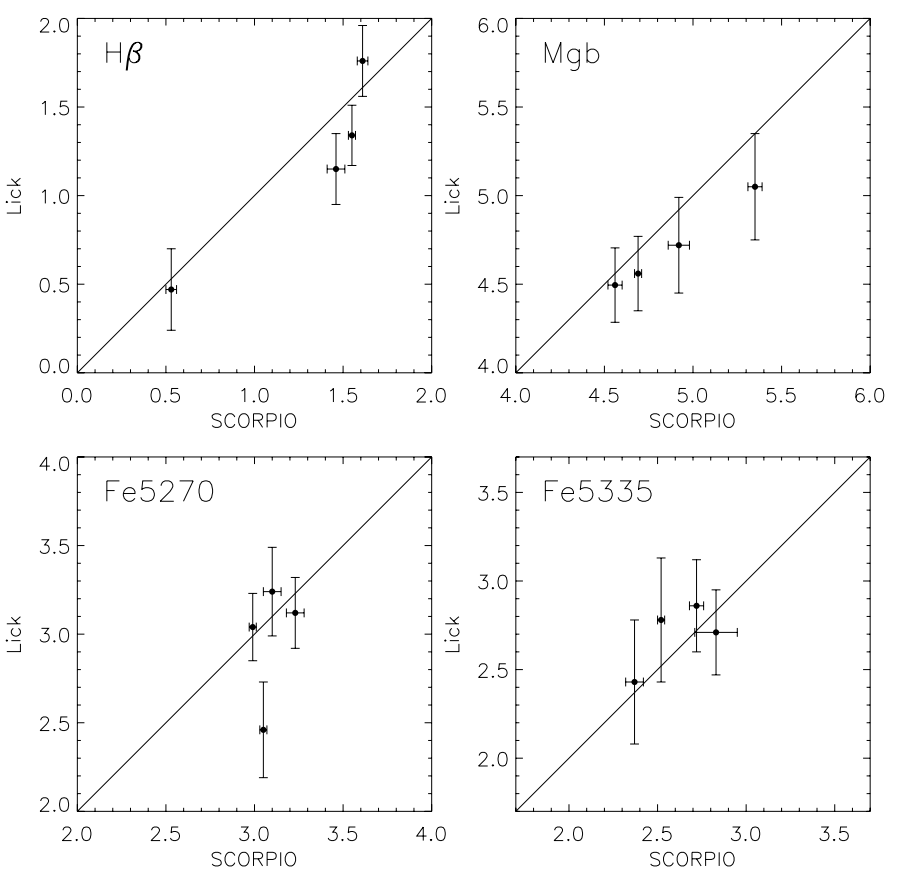

Fig. 2. The comparison of the calibrated absorption-line indices obtained with the spectrograph SCORPIO for the central parts $\left(1^{\prime \prime} \times 3^{\prime \prime} .5\right)$ of four galaxies and the standard Lick indices for them from the aperture spectroscopy of Trager et al. (1998) with the aperture of $2^{\prime \prime} \times 4^{\prime \prime}$.

we observed 22 stars from the list of Worthey et al. (1994) during several observational runs with the same grating and spectrograph setup; the comparison of the instrumental and standard Lick indices for these stars from the work of Worthey et al. (1994) is shown in Fig. 1. We calculated the linear regression formulae to transform our index measurements into the Lick system. The rms scatters of points near the linear dependencies derived are about $0.2 \AA$ for all 4 indices under consideration, within the observational errors of Worthey et al. (1994).

To correct the index measurements for the stellar velocity dispersion which is usually substantial in the centres of early-type galaxies, we smoothed the spectrum of the K3III standard star HD 167042 by a set of Gaussians of various widths, and the derived dependencies of index corrections on $\sigma$ were approximated by $4 \mathrm{~h}$-order polynomials and applied to the measured index values before their calibrations into the Lick system. Four galaxies of the five studied here have published central aperture index data obtained in the original Lick system (Trager et al. 1998). In Fig. 2 we compare our fully calibrated central index measurements averaged between $-2.2^{\prime \prime}$ and $+2.2^{\prime \prime}$ for every galaxy with the data of Trager et al. (1998) taking in mind their aperture of $2^{\prime \prime} \times 4^{\prime \prime}$. With 13 points of 16 within one sigma from the lines of equality, and with other 3 points within two sigma, the agreement seems to be good.

To evaluate the internal accuracy of our index measurements, we made some simulations of the deepest galactic spectrum, the one for NGC 2634. The high $S / N$ spectrum of the standard star HD 102328 was expanded along the slit with the normalisation corresponding to the galaxy fluxes observed at different distances from the centre, the Gaussian smoothing was applied to imitate a radial velocity dispersion distribution, the sky level and the noise observed were added, and after that our standard reduction procedure was applied to this artificial spectrum. By measuring the indices, we assured that (i) difficulties related to the sky subtraction affect only the index measurements in the 
very external parts of the index profiles, beyond $2 r_{\mathrm{e}}$. And (ii) the statistical accuracy of the indices estimated as the rms of the point-to-point scatter is $0.02-0.04 \AA$ over the range 0 to $0.2 r_{\mathrm{e}}$, $0.03-0.07 \AA$ over the range $0.2 r_{\mathrm{e}}$ to $0.5 r_{\mathrm{e}}, 0.10-0.15 \AA$ over the range $0.5 r_{\mathrm{e}}$ to $1 r_{\mathrm{e}}$, and $0.21-0.27 \AA$ over the range $1 r_{\mathrm{e}}$ to $2 r_{\mathrm{e}}$. As a visible illustration of the high internal accuracy of our index measurements, Fig. 3 compares two independent observations of NGC 3348, made in 2005 and in 2006 with the different slit orientations; the agreement of the indices is within $0.2 \AA$ up to $R=30^{\prime \prime}$, or $1.36 r_{\mathrm{e}}$.

\section{The results}

\subsection{Line-index profiles}

Figures 4 to 8 show the measured index profiles for all 5 galaxies under consideration. Two halves of the profiles are symmetrically reflected with respect to the centre and plotted by different signs. The first thing that is evident from Figs. 4 to 8 is that the index radial variations cannot be fitted by a single linear law over the full radius range, in opposition to well-established tradition. With our high index accuracy, we clearly see breaks, or changes in slopes, of the observed dependencies. Below we give brief comments for every galaxy.

NGC 2634, Fig. 4. This moderate-luminous elliptical galaxy was observed by us with the longest exposure of them all, so the observations are very deep. The index profiles are traced up to $3 r_{\mathrm{e}}$, though we suspect that the sky subtraction uncertainty may affect their shapes at $r>2 r_{\mathrm{e}}$. The emission lines are absolutely absent in the spectrum, and the $\mathrm{H} \beta$-index profile looks flat. The magnesium- and iron-index profiles decline with radius. Their behaviour with respect to the logarithm of the radius may be characterised as a sequence of linear pieces with different slopes. The points of slope breaks are close to $0.4 r_{\mathrm{e}}$, or $\log \left(r / r_{\mathrm{e}}\right)=-0.4$, and, less surely, to $0.8 r_{\mathrm{e}}$, or $\log \left(r / r_{\mathrm{e}}\right)=-0.1$. This break is seen in $\mathrm{Mgb}$ and $\mathrm{Fe} 5335$ profiles but not in the $\mathrm{Fe} 5270$ profile. We take the value of $r_{\mathrm{e}}$ for NGC 2634 from the work of Trujillo et al. (2004), where its photometric structure is described as a pure Sérsic (non-core) one. The galaxy is a member of large group dominated by a giant Sb-galaxy NGC 2550, but it is located rather far from the centre of the group and has its own subgroup consisting of the dwarf NGC 2634A and of the late-type barred spiral NGC 2633.

NGC 2810, Fig. 5. The observations are shallower reaching only $1.4 r_{\mathrm{e}}$. We determined the effective radius of the galaxy from our SCORPIO data in two ways: from the broad-band $V$-image that always precedes the spectral-mode exposure and from integration of the flux obtained in the spectral mode across the slit. The surface brightness profiles are well-fitted by a de Vaucouleurs' law (de Vaucouleurs 1948), and both approaches have consistently given $r_{\mathrm{e}}=14^{\prime \prime}$. The galaxy, though red and homogeneous photometrically, revealed strong emission lines in the spectrum, not only in the nucleus, but also over the full radial extension. Due to large difference between the velocity dispersion of stars and ionized gas in the nucleus, we succeeded in extracting a pure emission spectrum and estimated the ratio of the emission-line fluxes: $\mathrm{H} \beta /[\mathrm{O}$ III $] \lambda 5007=0.85$. This ratio is within the value interval found by Trager et al. (2000a) for their sample of elliptical galaxies, $0.33-1.25$, so we used it to correct the $\mathrm{H} \beta$ indices measured in the spectrum of NGC 2810 for the emission through calculating EW([O III] 25007 ) over the full radial extension along the slit and multiplying it by 0.85 . However, Fig. 5 presents the initial, uncorrected $\mathrm{H} \beta$ indices so the $\mathrm{H} \beta$ profile has a deep minimum in the centre of the galaxy.

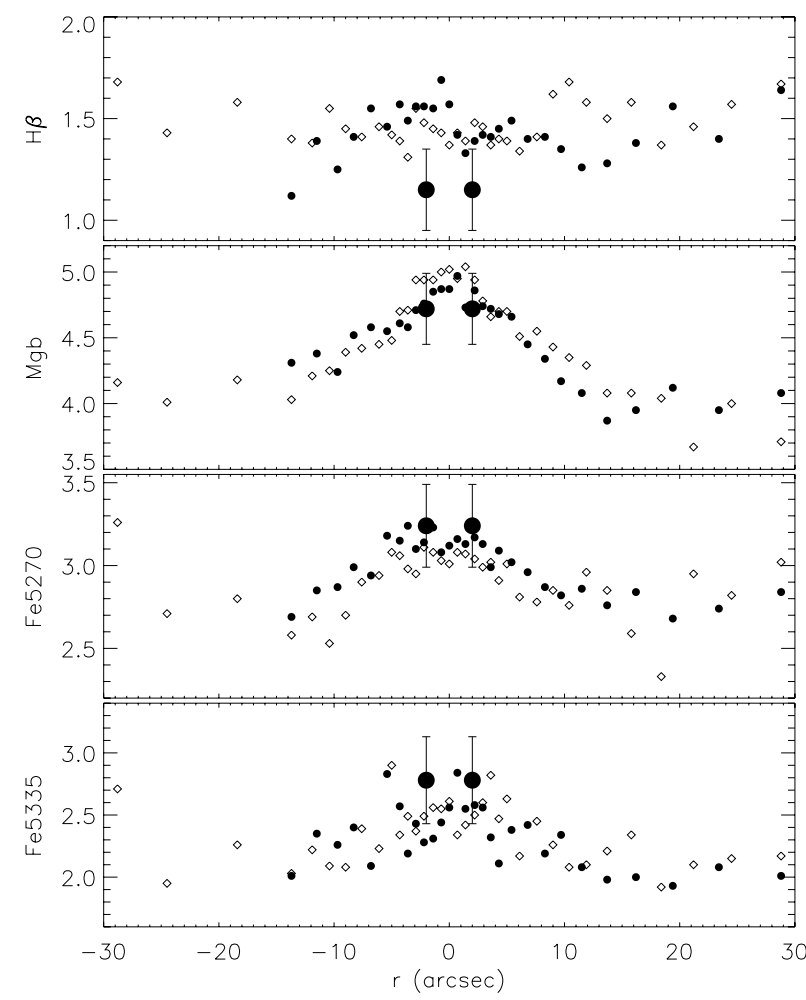

Fig. 3. Two independent observations of NGC 3348 in 2005 and in 2006 (small signs, filled and open) made with the orthogonal slit orientations. The aperture central measurements by Trager et al. (1998) are also shown by large filled circles with error bars.

The $\mathrm{Mgb}$ index, very high in the centre, falls steeply down to $\log \left(r / r_{\mathrm{e}}\right) \approx-0.1$ and stays roughly constant beyond this radius. The iron index decline is rather shallow over the full radius extension. The elliptical galaxy NGC 2810 is attributed neither by Garcia (1993) nor by Giuricin et al. (2000) to any galactic groups, so in our Table 1 we classify it formally as a field elliptical. But according to HYPERLEDA, a lot of small late-type galaxies are seen not far from NGC 2810; some of them are even caught by the SCORPIO field of view.

NGC 3348, Fig. 6. This galaxy is the most luminous in our sample, and it is the centre of its group. Trujillo et al. (2004) describe it as a core elliptical galaxy, and it is from this work that we take the effective radius value of $22^{\prime \prime}$. The spectrum demonstrates the weak emission line [O III $] \lambda 5007$. As we were not able to extract the pure emission line $\mathrm{H} \beta$, we will correct the index $\mathrm{H} \beta$ below for the emission in the statististical sense, by following the recommendation of Trager et al. (2000a): $\Delta \mathrm{H} \beta=0.6 \mathrm{EW}([\mathrm{O} \mathrm{III}] \lambda 5007)$. Figure 6 presents an uncorrected $\mathrm{H} \beta$ profile, as well as magnesium- and iron-index profiles and combines the data for two different exposures of the galaxy, that of March 2005 and that of April 2006 made with the orthogonal orientations of the slit. The metal-line profiles fall smoothly to $\log \left(r / r_{\mathrm{e}}\right) \approx-0.2$. Beyond this radius, the $\mathrm{Mg} \mathrm{b}$ and $\mathrm{Fe} 5335$ profiles look flat, and the Fe 5270 profile even rises. The final points of the profiles are around $r \approx 1.3 r_{\mathrm{e}}$. In April 2006, the faint galaxy at 2.5 to the east of NGC 3348, MCG +12-10-079, was also on the slit. It appeared to be the background early-type spiral galaxy, with the weak emission line [O III] $] \lambda 5007$ in the spectrum indicating $v_{r} \approx 10200 \mathrm{~km} \mathrm{~s}^{-1}$. Its nuclear Lick indices, $\mathrm{H} \beta=1.38, \mathrm{Mg} \mathrm{b}=3.32, \mathrm{Fe} 5270=3.03$, and $\mathrm{Fe} 5335=2.28$, are typical of the bulge of the moderately luminous Sb galaxy. 


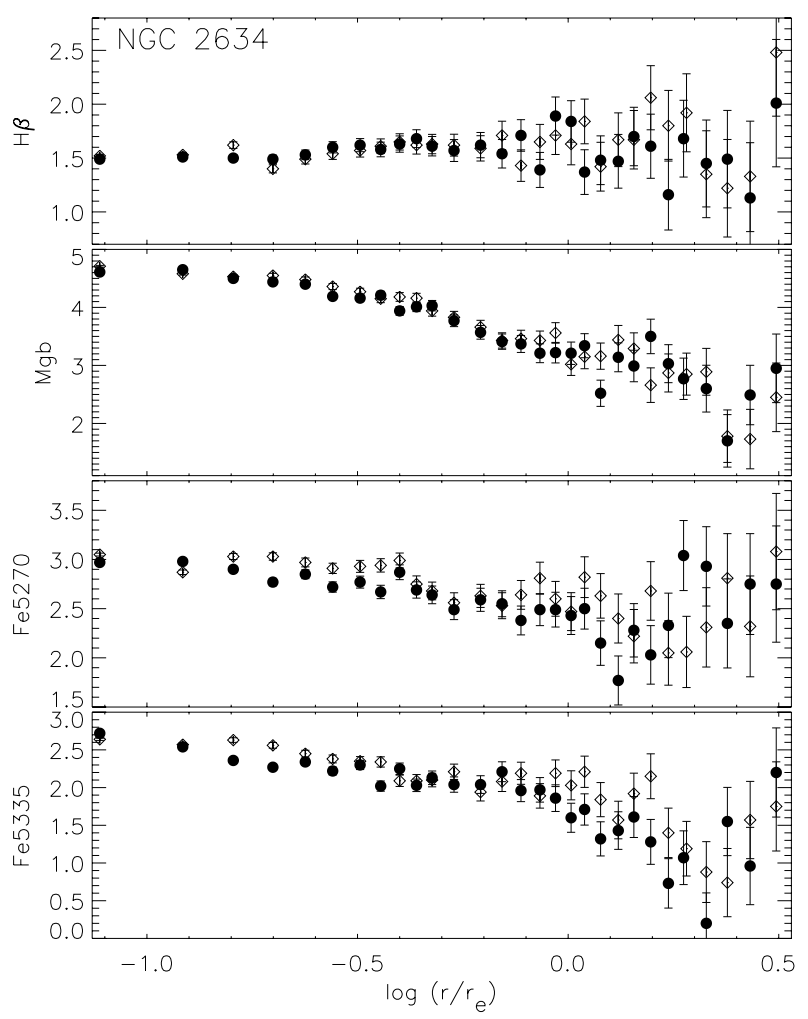

Fig. 4. The index profiles of NGC 2634.

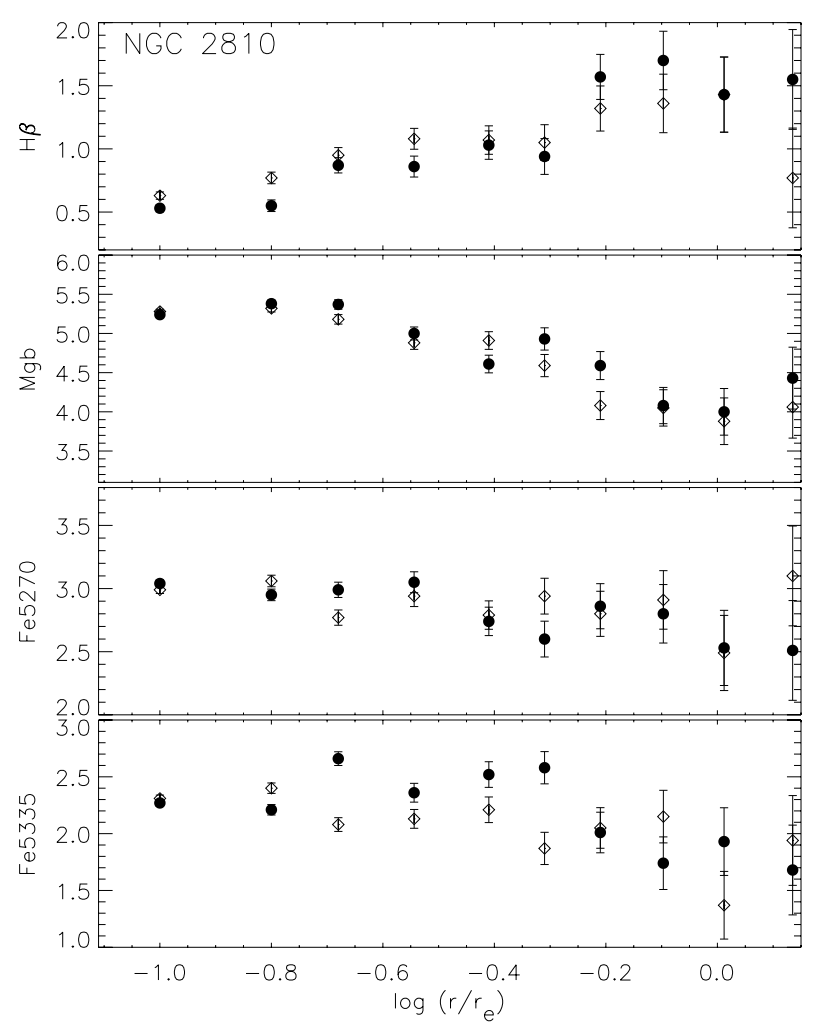

Fig. 5. The index profiles of NGC 2810.

NGC 4339, Fig. 7. This early-type galaxy is a Virgo member. It has a rather low luminosity for an elliptical and is classified as either E0 or S0 by different authors. We analysed its surface brightness profile obtained by tracing the flux integrated across the slit and have seen that the surface brightness profile beyond $r \approx 20^{\prime \prime}$ is well-fitted by an exponential, so beyond

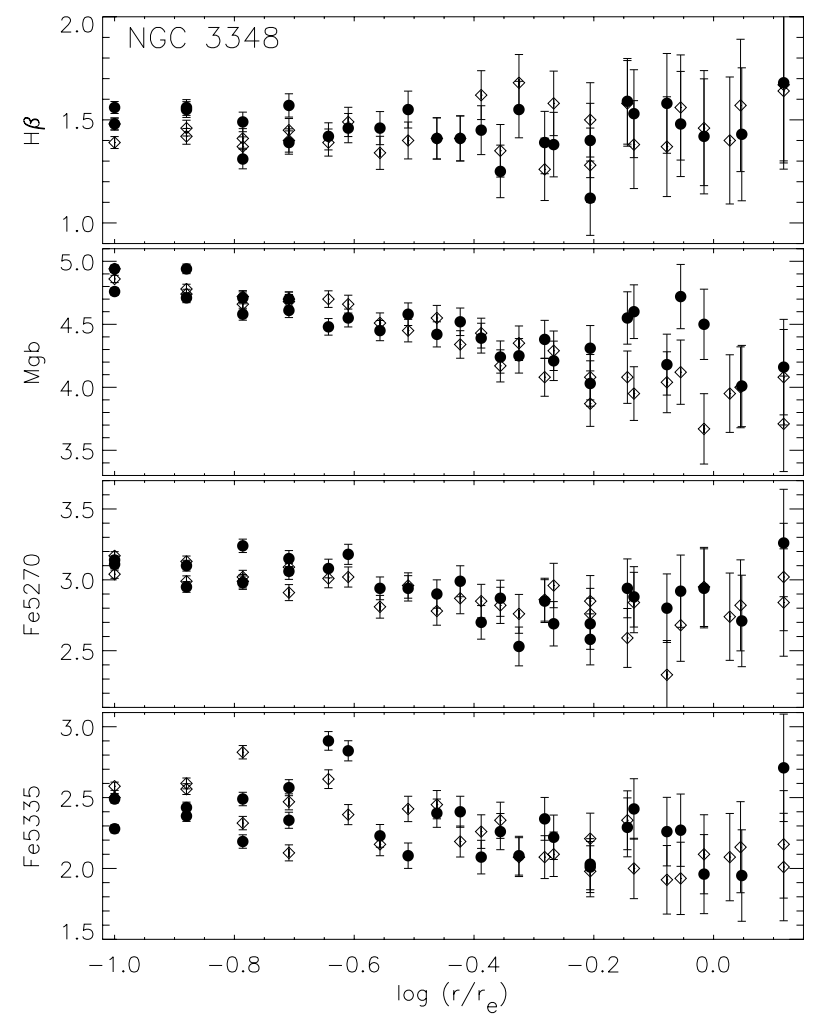

Fig. 6. The index profiles of NGC 3348.

$r \approx 20^{\prime \prime}$ the stellar disk dominates. But within this radius we see a de Vaucouleurs' bulge with $r_{\mathrm{e}}=26^{\prime \prime}$, so this effective radius is used to parametrise the index profiles in Fig. 7. Caon et al. (1994) give an effective radius of $277^{\prime \prime} 7$ along the major and 25".8 along the minor axes, so in agreement with our measurements. Because of the bad weather, the exposure of NGC 4339 was short, and we only traced the index profiles for this galaxy to $0.5 r_{\mathrm{e}}$. Nevertheless even these short profiles revealed the breaks of slopes, this time around $\log \left(r / r_{\mathrm{e}}\right) \approx$ $-0.7-0.6$. The emission is absent in the galaxy, so the break in the $\mathrm{H} \beta$ profile at $\log \left(r / r_{\mathrm{e}}\right) \approx-0.7$ reflects the real change of the mean stellar age.

IC 2179, Fig. 8. This elliptical galaxy, being the fainter neighbour of a giant spiral galaxy NGC 2347, has not been studied in detail to now. In particular, the close bright star might preclude any attempts at surface photometry. We checked the brightness profile along the slit of the spectrograph and ensured that two halves of the profile diverge at $r>30^{\prime \prime}$. However, the surface brightness profile is very good inside this radius and can be fitted by a de Vaucouleurs' law (de Vaucouleurs 1948) with $r_{\mathrm{e}} \approx 18^{\prime \prime}$; if we take only the "fainter" half of the profile, we obtain $r_{\mathrm{e}} \approx 15^{\prime \prime}$ in the radius range between $5^{\prime \prime}$ and $56^{\prime \prime}$ - this value is used to parametrise the profiles in Fig. 8. The metalline profiles show two breaks - at $\log \left(r / r_{\mathrm{e}}\right) \approx-0.3-0.2$ and at $\log \left(r / r_{\mathrm{e}}\right) \approx 0-+0.1$; the $\mathrm{H} \beta$ index profile only reveals the latter break. The emission lines are absent in the spectrum, so the $\mathrm{H} \beta$ index measurements are reliable.

\subsection{The magnesium-to-iron ratios}

Figure 9 presents the magnesium-iron diagrams for the index profiles given in Figs. 4-8. We compare our observational data with the evolutionary-synthesis models for SSP of Thomas et al. (2003), which were calculated for several values of magnesiumto-iron abundance ratio, namely, the $0.5,1,2$, and 3 solar ones 


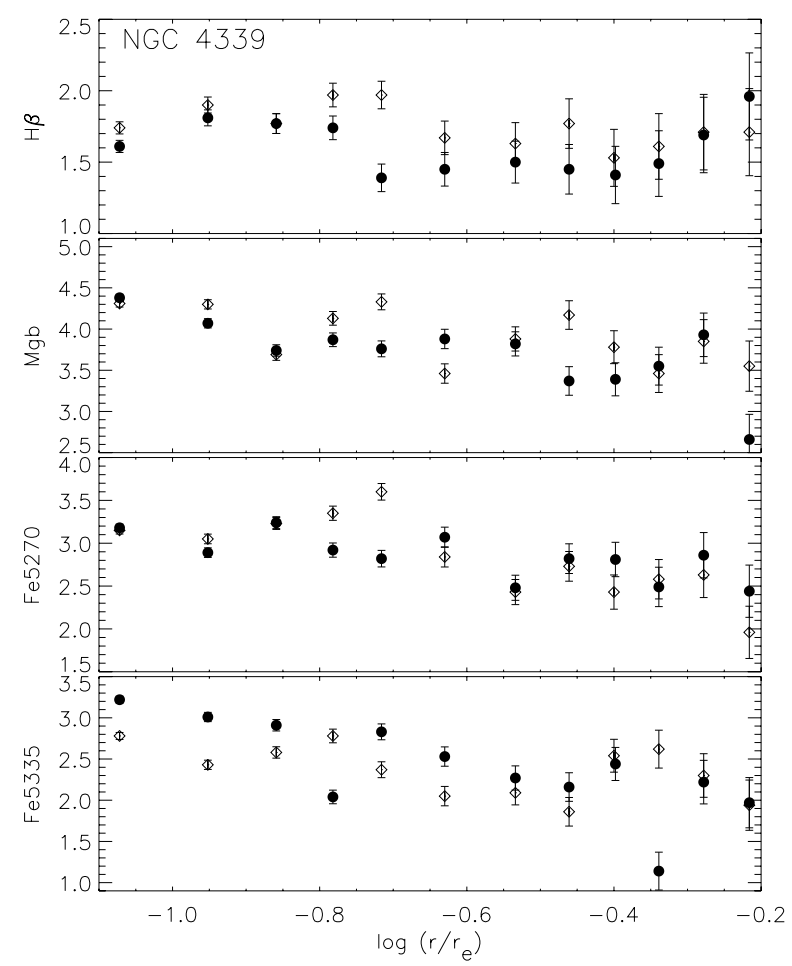

Fig. 7. The index profiles of NGC 4339.

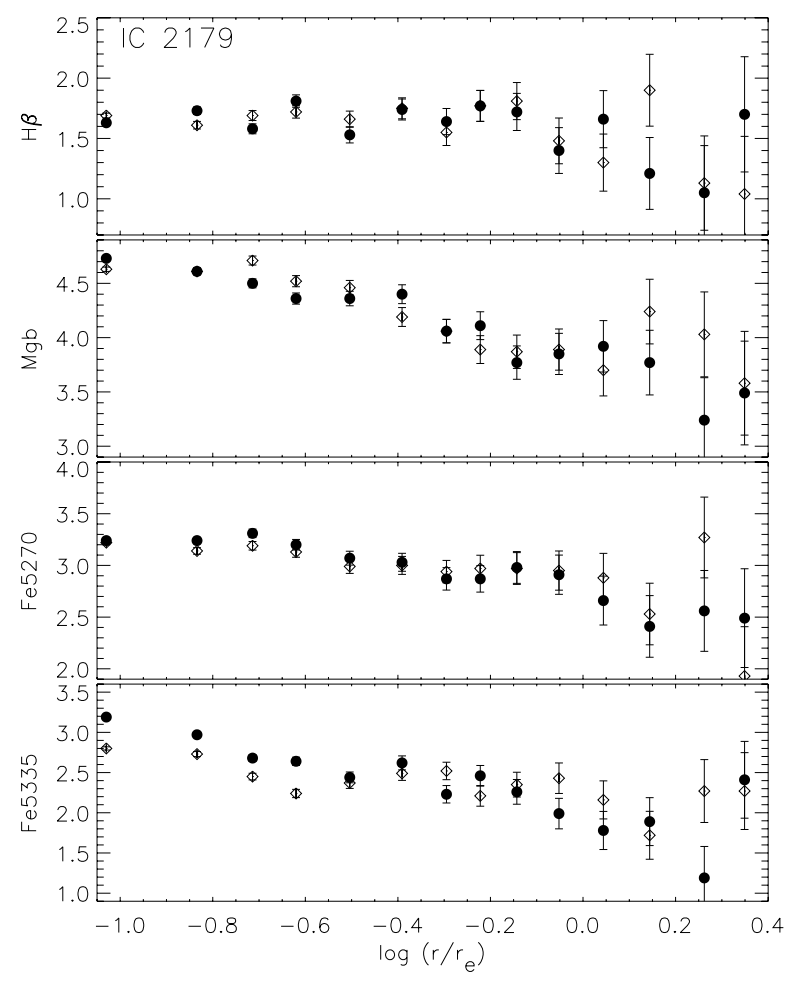

Fig. 8. The index profiles of IC 2179 .

$([\mathrm{Mg} / \mathrm{Fe}]=-0.3,0.0,+0.3$, and +0.5$)$. As it is well known elliptical galaxies have magnesium-overabundant stellar populations. Our results for the magnesium-to-iron ratio in the galaxies under consideration agree with all previous studies of the stellar population in elliptical galaxies. The mean values of $[\mathrm{Mg} / \mathrm{Fe}]$ ratio change from +0.1 in NGC 4339 to +0.3 in NGC 3348, and our galaxies demonstrate clear dependence of the magnesium overabundance on the stellar velocity dispersion. As already noted by Trager et al. (2000b), the more massive elliptical galaxies have higher magnesium-to-iron ratio. Within individual galaxies, the $[\mathrm{Mg} / \mathrm{Fe}]$ ratios remain roughly constant along the radius as noted earlier by Worthey, et al. (1992), Davies, et al. (1993), and Fisher et al. (1995). The exception is NGC 2810 where the centre has almost $[\mathrm{Mg} / \mathrm{Fe}]=+0.5-$ too high for its stellar velocity dispersion - and toward the outer part this ratio falls to its normal value, about +0.3 . This elliptical galaxy has the most prominent emission lines in the spectrum; and as we see below, the young age of its central part is evidence of the secondary star formation burst perhaps provoked by a minor merger. Less certain change of $[\mathrm{Mg} / \mathrm{Fe}]$ from +0.1 to +0.2 can be noticed along the radius in NGC 4339.

\subsection{The ages and metallicities along the radius}

In this subsection we present so-called SSP-equivalent ages and metallicities of the stellar populations determined by fitting the observed Lick indices $\mathrm{H} \beta, \mathrm{Mg}$ b, Fe 5270 , and Fe 5335 by oneburst evolutionary synthesis models. The SSP models assume that all the stars within a stellar system have the same age and metallicity. In fact, the galaxies may be composite stellar systems formed during several star formation bursts, with a different chemical composition in general. We therefore determined some average stellar ages and metallicities. The recent simulations by Serra \& Trager (2006) have shown that the metallicities determined in this way are close to luminosity-weighted average metallicities, while the SSP-equivalent age is always biased toward the age of the youngest stars within the stellar system and is somewhere between the luminosity-weighted average age and that of the youngest stars.

We consider together the $\mathrm{H} \beta$ absorption-line index and the metal-line combined one,

$[\mathrm{MgFe}] \equiv \sqrt{\mathrm{Mgb}\langle\mathrm{Fe}\rangle}$,

where

$\langle\mathrm{Fe}\rangle \equiv \frac{\mathrm{Fe} 5270+\mathrm{Fe} 5335}{2}$,

to disentangle the age and metallicity effects and to determine both characteristics simultaneously. The model calculations show that, at the diagram $\mathrm{H} \beta$ vs. $[\mathrm{MgFe}]$, the line families of equal metallicities and of equal ages (Figs. 10) have different slopes, so representing a somewhat curved coordinate frame. By using the model grid by Thomas et al. (2003) for $[\mathrm{Mg} / \mathrm{Fe}]=+0.3$, we applied a $2 \mathrm{D}$ interpolation between the discrete model nodes to calculate the mean luminosity-weighted stellar ages and metallicities for each point of the profiles of Figs. 4-8. The H $\beta$ indices in NGC 2810 and NGC 3348, where we see a significant emission contribution, are corrected for the emission by using the measurements of the equivalent width for the emission line [O III] $\lambda 5007$ : the additive correction is obtained by multiplying EW([O III] 25007$)$ by 0.85 for NGC 2810 (the individual coefficient estimated from the SCORPIO spectra) and by 0.6 for NGC 3348 (the statistical coefficient from Trager et al. 2000a).

To extract main tendencies of age and metallicity variations along the radius, we go on to average the indices, age, and metallicity estimates over wider spatial bins near some key points of the profiles: $r_{\mathrm{e}} / 8, r_{\mathrm{e}} / 5, r_{\mathrm{e}} / 2, r_{\mathrm{e}}, 1.3 r_{\mathrm{e}}$, and $2 r_{\mathrm{e}}$. Here we calculate the errors of the mean indices and those of the mean ages and metallicities as simple point-to-point scatter divided by the number of measurements per bin. The results of this procedure are given in Table 3 and shown in Fig. 10 for each galaxy. 

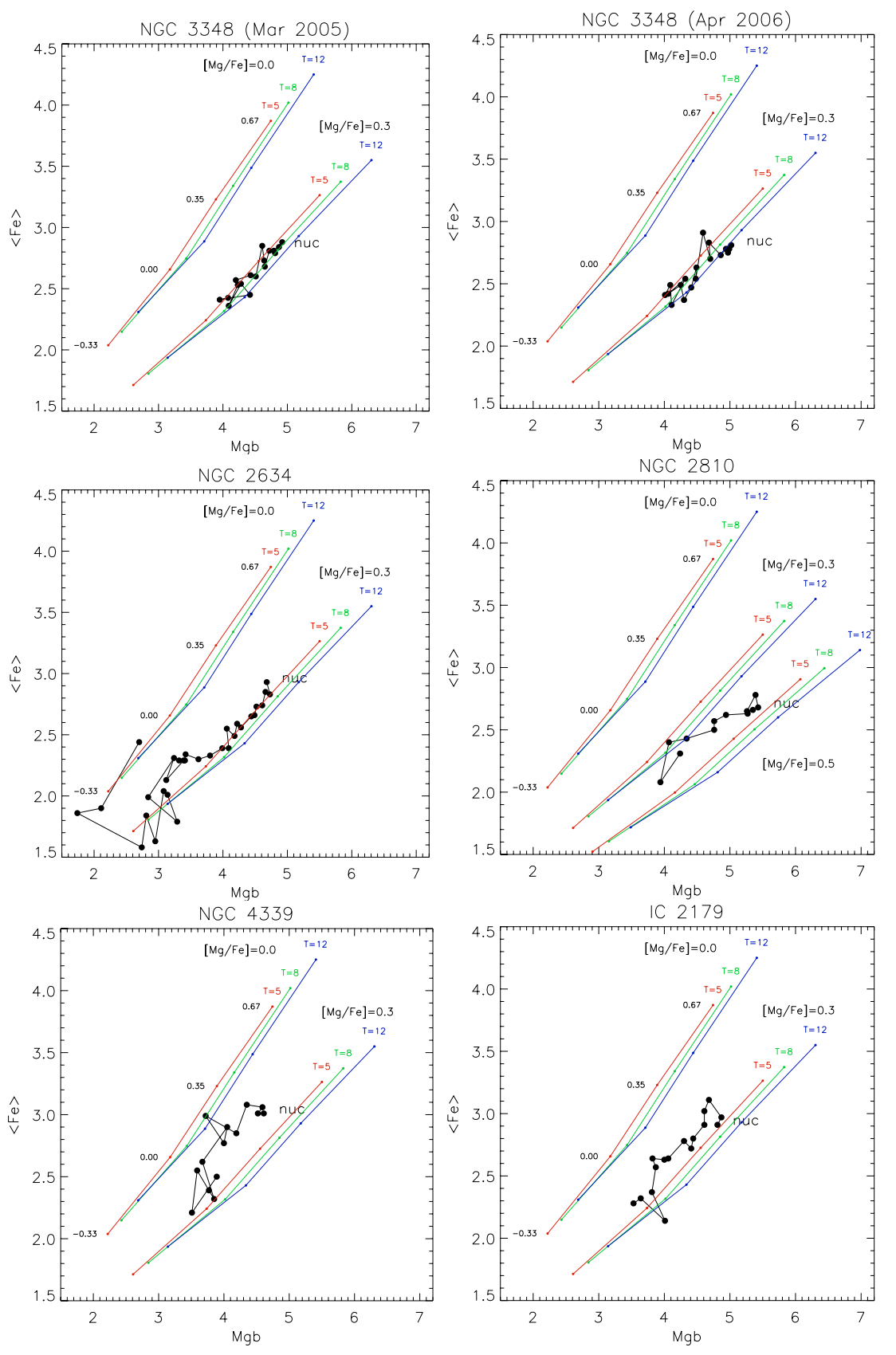

Fig. 9. The magnesium-iron index diagrams for all the galaxies under consideration, with the Lick indices averaged over both sides of the slit taken along the radius with the steps as in Figs. 4-8 (circles connected by solid black lines, the nucleus marked as "nuc"). The SSP models of Thomas et al. (2003) for $[\mathrm{Mg} / \mathrm{Fe}]=0.0,+0.3$, and +0.5 (the last for NGC 2810 only) are plotted as a reference frame; the solid coloured lines represent stellar population models of equal ages; the metallicities for the models are $+0.67,+0.35,0.0,-0.33$ from the top to bottom of each line.
Below we discuss the stellar metallicity and age variations along the radius in the individual galaxies.

NGC 2634, Fig. 10a. This only galaxy in our sample has no stellar age gradient along the radius. The stellar population is old everywhere, 10-13 Gyr, and the mean metallicity changes from roughly +0.2 dex in the nucleus to less than -0.4 dex at $r>r_{\mathrm{e}}$. It may seem that the outer measurements of the indices are outside the parameter range of the models. But this discordance only reflects the need for more precision in treating low metallicities by Thomas et al. (2003) (and by any other model sets as well). For comparison, we have plotted the index measurements for the Galactic globular clusters NGC 6624, NGC 6838, NGC 6356, and NGC 6539 with $[\mathrm{Fe} / \mathrm{H}]$ from -0.35 to -0.66 from Beasley et al. (2004); they are all near the outer measurements for NGC 2634 and outside the model grid of Thomas et al. (2003).

NGC 2810, Fig. 10b. The stellar population in the centre of this field elliptical is not very old, with a mean age of only
5-6 Gyr. The metallicity of stars in the centre is very high, the highest in our sample (though the galaxy is not the most massive and luminous one). Beyond the radius of $3^{\prime \prime}$ to $5^{\prime \prime}$, the stellar population is homogeneously old, about $10 \mathrm{Gyr}$, and the metallicity is approximately solar. As we have also seen, there is a sharp increase in $[\mathrm{Mg} / \mathrm{Fe}]$ ratio in the nucleus of NGC 2810 , so by taking a considerable amount of ionized gas in this galaxy into account, we may conclude that a minor merger has taken place some billion years ago to have provoked a short, intense nuclear star formation burst that increased the mean metallicity and decreased the mean age of stars.

NGC 3348, Fig. 10c. The galaxy was classified by RSA as a regular E0, but it has now shown itself to be very unusual. The radial variations of the mean stellar age in this galaxy look like a mirror of NGC 2810: the nucleus is old, and the stars become younger and younger along the radius becoming 3-4 Gyr old at $r>r_{\mathrm{e}}$. The metallicity gradient, though negative at $r=0-0.5 r_{\mathrm{e}}$, becomes positive in the radius range 
Table 3. The mean indices, ages, and metallicities along the radius in the galaxies of our sample.

\begin{tabular}{|c|c|c|c|c|c|c|}
\hline Galaxy & $\log \left(r / r_{\mathrm{e}}\right)$ & $\mathrm{H} \beta$ & $\mathrm{Mg} \mathrm{b}$ & $\langle\mathrm{Fe}\rangle$ & {$[\mathrm{Z} / \mathrm{H}]$} & Age [Gyr] \\
\hline NGC 2634 & $\begin{array}{c}-\infty \\
-0.8 \\
-0.3 \\
0.0 \\
0.3 \\
0.45\end{array}$ & $\begin{array}{l}1.55 \pm 0.02 \\
1.51 \pm 0.03 \\
1.61 \pm 0.01 \\
1.71 \pm 0.08 \\
1.60 \pm 0.13 \\
1.74 \pm 0.31\end{array}$ & $\begin{array}{l}4.69 \pm 0.02 \\
4.54 \pm 0.03 \\
3.89 \pm 0.06 \\
3.25 \pm 0.08 \\
2.78 \pm 0.06 \\
2.40 \pm 0.25\end{array}$ & $\begin{array}{l}2.86 \pm 0.03 \\
2.69 \pm 0.05 \\
2.36 \pm 0.03 \\
2.24 \pm 0.08 \\
1.71 \pm 0.12 \\
2.17 \pm 0.16\end{array}$ & $\begin{array}{c}+0.25 \pm 0.02 \\
+0.13 \pm 0.04 \\
-0.11 \pm 0.02 \\
-0.17 \pm 0.03 \\
-0.5 \pm ? \\
-0.5 \pm ?\end{array}$ & $\begin{array}{c}11.2 \pm 0.6 \\
12.5 \pm 0.8 \\
13.3 \pm 0.6 \\
9.5 \pm 0.7 \\
11 \pm ? \\
-\end{array}$ \\
\hline NGC 2810 & $\begin{array}{c}-\infty \\
-0.7 \\
-0.3 \\
0.0 \\
0.135\end{array}$ & $\begin{array}{c}1.70 \pm 0.03 \\
1.79 \pm 0.07 \\
1.61 \pm 0.09 \\
1.82 \pm 0.05 \\
1.62 \pm 0.4\end{array}$ & $\begin{array}{c}5.35 \pm 0.04 \\
5.19 \pm 0.08 \\
4.73 \pm 0.08 \\
4.00 \pm 0.04 \\
4.24 \pm 0.2\end{array}$ & $\begin{array}{c}2.71 \pm 0.02 \\
2.63 \pm 0.06 \\
2.50 \pm 0.04 \\
2.24 \pm 0.12 \\
2.31 \pm 0.2\end{array}$ & $\begin{array}{c}+0.47 \pm 0.06 \\
+0.45 \pm 0.03 \\
+0.10 \pm 0.04 \\
-0.04 \pm 0.10 \\
+0.1 \pm ?\end{array}$ & $\begin{array}{c}6.5 \pm 1.3 \\
5.3 \pm 1.0 \\
11.2 \pm 1.8 \\
8.8 \pm 1.5 \\
12 \pm 7\end{array}$ \\
\hline NGC 3348 & $\begin{array}{c}-\infty \\
-0.9 \\
-0.6 \\
-0.3 \\
0.0 \\
+0.12\end{array}$ & $\begin{array}{l}1.46 \pm 0.05 \\
1.63 \pm 0.02 \\
1.72 \pm 0.04 \\
1.72 \pm 0.06 \\
1.90 \pm 0.01 \\
2.06 \pm 0.02\end{array}$ & $\begin{array}{l}4.92 \pm 0.06 \\
4.78 \pm 0.05 \\
4.57 \pm 0.03 \\
4.21 \pm 0.04 \\
4.12 \pm 0.10 \\
4.00 \pm 0.08\end{array}$ & $\begin{array}{c}2.81 \pm 0.06 \\
2.77 \pm 0.02 \\
2.73 \pm 0.07 \\
2.46 \pm 0.04 \\
2.44 \pm 0.02 \\
2.61 \pm 0.2\end{array}$ & $\begin{array}{l}+0.30 \pm 0.02 \\
+0.29 \pm 0.02 \\
+0.26 \pm 0.04 \\
+0.04 \pm 0.01 \\
+0.10 \pm 0.04 \\
+0.24 \pm 0.05\end{array}$ & $\begin{array}{c}10.6 \pm 0.3 \\
9.2 \pm 0.6 \\
8.0 \pm 1.0 \\
9.6 \pm 1.0 \\
6.6 \pm 0.4 \\
4 \pm ?\end{array}$ \\
\hline NGC 4339 & $\begin{array}{l}-\infty \\
-0.9 \\
-0.7 \\
-0.5 \\
-0.3\end{array}$ & $\begin{array}{l}1.61 \pm 0.03 \\
1.81 \pm 0.03 \\
1.70 \pm 0.10 \\
1.59 \pm 0.07 \\
1.70 \pm 0.06\end{array}$ & $\begin{array}{l}4.56 \pm 0.04 \\
3.95 \pm 0.14 \\
3.90 \pm 0.12 \\
3.81 \pm 0.16 \\
3.50 \pm 0.18\end{array}$ & $\begin{array}{l}3.03 \pm 0.09 \\
2.92 \pm 0.07 \\
2.77 \pm 0.10 \\
2.36 \pm 0.05 \\
2.26 \pm 0.13\end{array}$ & $\begin{array}{l}+0.34 \pm 0.04 \\
+0.14 \pm 0.04 \\
+0.14 \pm 0.16 \\
-0.12 \pm 0.06 \\
-0.19 \pm 0.05\end{array}$ & $\begin{array}{c}9.1 \pm 1.0 \\
7.1 \pm 0.7 \\
7.5 \pm 2.3 \\
11.3 \pm 2.0 \\
11.3 \pm 0.9\end{array}$ \\
\hline IC 2179 & $\begin{array}{c}-\infty \\
-0.7 \\
-0.3 \\
0.0 \\
+0.3\end{array}$ & $\begin{array}{l}1.64 \pm 0.02 \\
1.69 \pm 0.03 \\
1.70 \pm 0.04 \\
1.46 \pm 0.08 \\
1.23 \pm 0.16\end{array}$ & $\begin{array}{l}4.78 \pm 0.02 \\
4.55 \pm 0.05 \\
4.12 \pm 0.07 \\
3.84 \pm 0.05 \\
3.58 \pm 0.16\end{array}$ & $\begin{array}{c}3.02 \pm 0.03 \\
2.91 \pm 0.06 \\
2.68 \pm 0.04 \\
2.47 \pm 0.10 \\
2.30 \pm 0.2\end{array}$ & $\begin{array}{c}+0.44 \pm 0.02 \\
+0.33 \pm 0.03 \\
+0.10 \pm 0.04 \\
-0.05 \pm 0.08 \\
-0.13 \pm ?\end{array}$ & $\begin{array}{c}7.0 \pm 0.6 \\
7.8 \pm 0.8 \\
9.5 \pm 1.0 \\
12.2 \pm 0.4 \\
12 \pm ?\end{array}$ \\
\hline
\end{tabular}

of $0.5 r_{\mathrm{e}}-1.3 r_{\mathrm{e}}$. Since NGC 3348 also shows extended emission lines in the spectrum, we may suggest that the galaxy has also experienced a minor merger. But this time the satellite involved had a smaller amount of gas than that of NGC 2810. As a result, no nuclear star formation burst has been provoked, but the contribution of young and metal-rich stars of the merged galaxy is clearly seen in the outer part of NGC 3348. As evidence in favour of our hypothesis of a minor merger for NGC 3348, we demonstrate here the colour map of the galaxy (Fig. 11). We retrieved the HST/ACS images of NGC 3348 obtained through two different filters, F435W and F814W, on January 20, 2003, in the framework of the programme by Harris on globular cluster systems in early-type galaxies (Proposal ID 9427). By dividing one image by another and by taking 2.5 logarithms of the partial, we obtained the colour map, close to $B-I$, that is presented in Fig. 11. One can see a lot of thin red filaments irregularly distributed over the whole extension of the galaxy. Evidently, this "prototype of E0", as Sandage \& Bedke (1994) characterized NGC 3348 in their Atlas, contains in fact a noticeable amount of diffuse dust and ionized gas coupled with this dust, which may be accreted rather recently.

NGC 4339, Fig. 10d. Though less prominent than NGC 2810, NGC 4339 has also shown some signs of the circumnuclear young metal-rich stellar disk that may be a product of the secondary nuclear star formation burst. However, the gas is absent in this galaxy.

IC 2179, Fig. 10e. In IC 2179 the nucleus is also younger than the outer part; but unless NGC 2810 and NGC 4339, IC 2179 does not seem to have a young circumnuclear stellar disk producing a "step" in age radial variations in the former galaxies. Instead we observe the gradual smooth age increase along the radius, from 8 Gyr to $\sim 15$ Gyr toward $2 r_{\mathrm{e}}$. The metallicity decrease along the radius is also very smooth, since IC 2179 is the only galaxy in our sample where we do not see flattening of the metallicity gradient at $r>0.5 r_{\mathrm{e}}$.

\section{Discussion}

The main point of our discussion concerns the metallicity gradients in elliptical galaxies, which are one of the signatures of their origin. As already mentioned, the monolithic dissipative collapse of a protogalactic cloud produces steep stellar metallicity gradients along the radius: from -0.5 (Carlberg 1984) to -1.0 dex per radius dex (Kobayashi 2004). As the mean metallicity gradients obtained to date under the assumption of the constant stellar age along the radius from the colour and metal-line index gradients was about -0.2 dex per radius dex, the opinion has formed that a major merger is typical of a luminous elliptical galaxy to flatten its metallicity gradient. Refining this zero-order approach, Kobayashi (2004), Forbes et al. (2005), and Ogando et al. (2005) all note a wide spread of metallicity gradients just for luminous (most massive) elliptical galaxies, thereby suggesting that there is a variety of evolutionary histories for elliptical galaxies, from quasi-monolithic collapse (or early gas-rich multiple mergers) to "dry" major mergers.

In our work, we have disentangled the age and metallicity effects and measured both characteristics of the stellar populations as they change along the radius. First of all, in most galaxies the stellar age is not constant along the radius: its behaviour is quite various and does not depend on the galaxy's luminosity or environment. Secondly, when we have carefully analysed the metallicity profiles over the large radial base, to at least $1.3 r_{\mathrm{e}}$, 

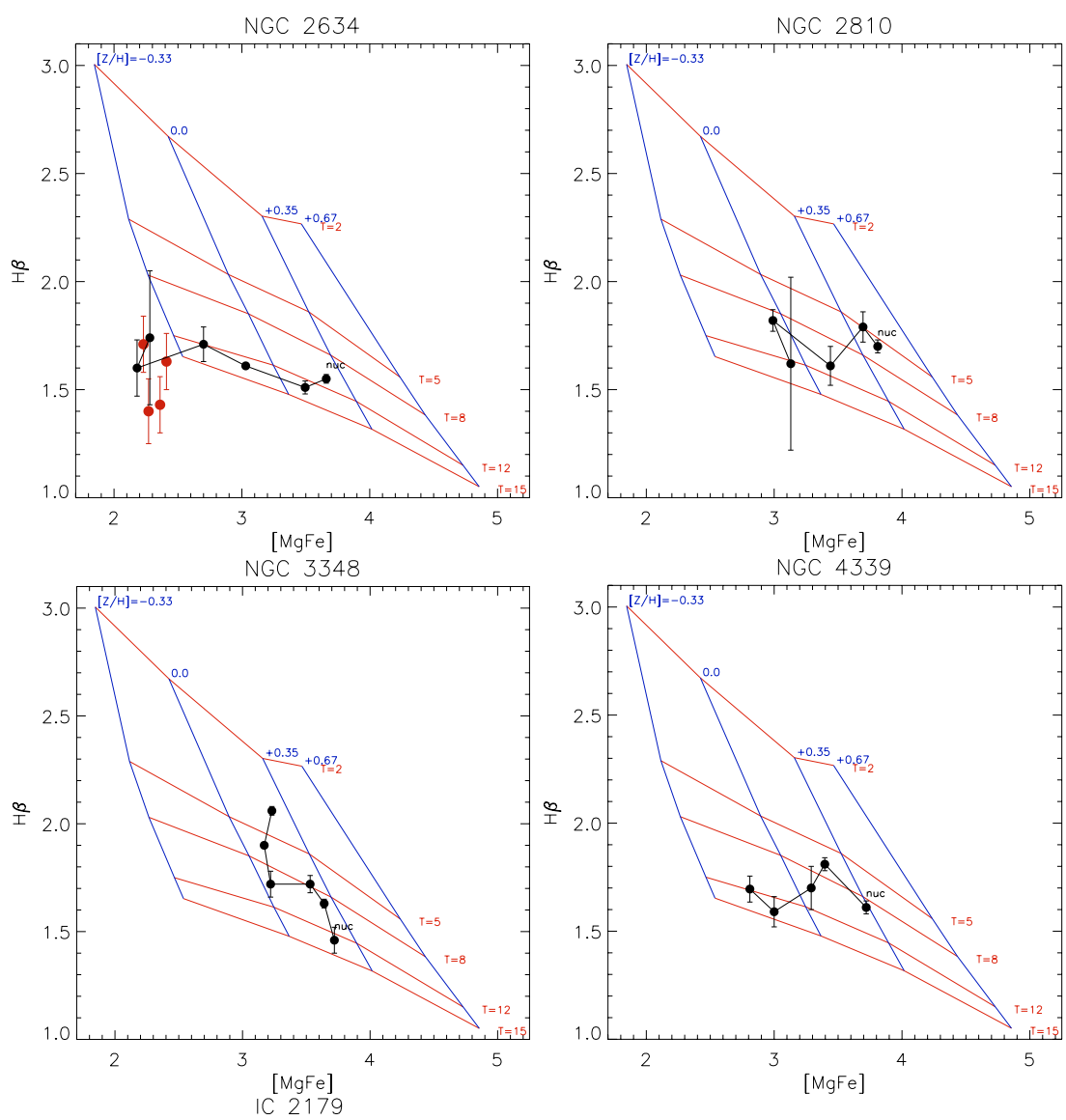

Fig. 10. The combined metal-line index vs. $\mathrm{H} \beta$ diagrams for all the galaxies under consideration with the Lick indices averaged in wide bins near the key points along the radius - see the text and the Table 3 (circles with error bars, the nucleus being marked as "nuc"). The SSP models of Thomas et al. (2003) for $[\mathrm{Mg} / \mathrm{Fe}]=+0.3$ are plotted as a reference frame; thin red and blue lines represent sequences of equal ages and metallicities, correspondingly. At the diagram for NGC 2634 several globular clusters of our Galaxy are also plotted as large red circles. we find that the metallicity variations cannot be described by a single power law over the full radial extension in most galaxies. The break of the slope is evident at $\sim 0.5 r_{\mathrm{e}}$ the outer parts showing the flatter metallicity gradients. We calculated the metallicity gradients for our 5 galaxies separately within $r<0.5 r_{\mathrm{e}}$ and in the radius range of $0.5 r_{\mathrm{e}}-1 r_{\mathrm{e}}$, and the results are given in Table 4 with the galaxies ranked according to their stellar velocity dispersion (mass).

From Table 4 one can see that the metallicity gradients in the inner parts of the galaxies are rather steep, steeper than the -0.4 dex per radius dex, and are consistent with the model of monolithic dissipative collapse. In the outer parts, the metallicity gradients are consistent with being zero; also taking the very inhomogeneous mean age distributions at $r>0.5 r_{\mathrm{e}}$ into account, we may suggest that the outer parts of the elliptical galaxies have been transformed in a secular manner through minor mergers, not by a major merger. This picture differs strongly from the conventional scenario of elliptical galaxy formation.
Table 4. Metallicity gradients in our galaxies.

\begin{tabular}{ccc}
\hline \hline Galaxy & $\begin{array}{c}\mathrm{d}[\mathrm{Z} / \mathrm{H}] / \mathrm{d} \log r \\
\left(r<0.5 r_{\mathrm{e}}\right)\end{array}$ & $\begin{array}{c}\mathrm{d}[\mathrm{Z} / \mathrm{H}] / \mathrm{d} \log r \\
\left(0.5 r_{\mathrm{e}}<r<r_{\mathrm{e}}\right)\end{array}$ \\
\hline NGC 3348 & $-0.42 \pm 0.05$ & $+0.20 \pm 0.17$ \\
NGC 2810 & $-0.88 \pm 0.17$ & $-0.47 \pm 0.47$ \\
IC 2179 & $-0.58 \pm 0.18$ & $-0.50 \pm 0.40$ \\
NGC 2634 & $-0.52 \pm 0.16$ & $-0.20 \pm 0.17$ \\
NGC 4339 & $-0.82 \pm 0.52$ & - \\
\hline
\end{tabular}

An interesting question is the possible connection between metallicity and age variations. Earlier, Henry \& Worthey (1999) noted an anticorrelation between the metallicity and age gradients found over the galaxy sample typically measured to $0.5-1 r_{\mathrm{e}}$. The slope of this relation appeared to be close to $-\frac{3}{2}$, which was a ratio of the sensitivities of the majority of metal-line indices to the age and metallicity variations, $\Delta \mathrm{Age} / \Delta \mathrm{Z}$ (Worthey 1994). Henry \& Worthey (1999) propose that (i) all the differences between the measured metallicity and age gradients in 


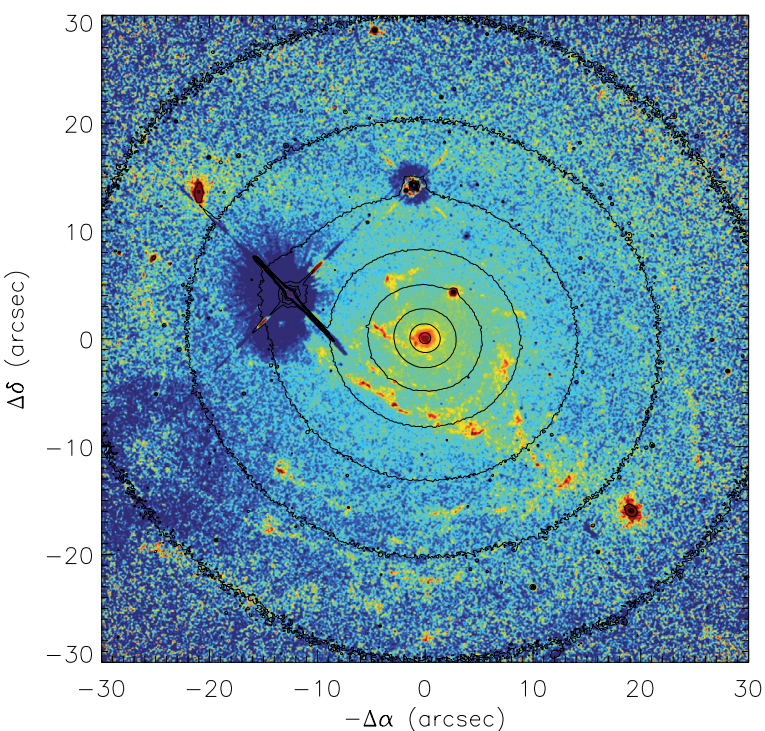

Fig. 11. The colour $F 435 W-F 814 W$ (HST/ACS) map of NGC 3348.

elliptical galaxies are due to random and systematic errors in index evaluation, producing the correlated scatter of the measured metallicity and age gradients and (ii) that, in fact, all the elliptical galaxies have to have exactly $\mathrm{d}[\mathrm{Z} / \mathrm{H}] / \mathrm{d} \log r=-0.25$ and $\mathrm{d} \log T / \mathrm{d} \log r=+0.1$. The accuracy of our measurements of the Lick indices along the radius of elliptical galaxies is higher than earlier results. We are therefore confident that the difference between the inner and outer metallicity gradients, say, in NGC 3348 or NGC 2634, is statistically significant. But indeed, in our sample too, the metallicity and age gradients show a certain anticorrelation (if we exclude the very outer part of NGC 2810), with a slope of $-1.20 \pm 0.32$. This is coincident with $-\frac{3}{2}$ within the error. Although this correlation may be real, its physical origin remains uncertain. A possible qualitative explanation is that additional star formation would simultaneously produce additional metals, while lowering the mean stellar age.

Another important point concerning the observational signatures of the dominant mechanism for elliptical galaxy formation is correlation between the dynamical parameters, mass or escape velocity, and the mean abundance characteristics of the stellar population. Initially, the well-known correlation between luminosity and the colour of elliptical galaxies was treated as mass-metallicity relation (Faber 1973) similar to what is known for other types of galaxies (e.g. Pilyugin, et al. 2004). But central-aperture spectral investigations of complete samples of nearby elliptical galaxies have given somewhat unexpected results: when disentangling the age-metallicity degeneracy and simultaneously measuring age, metallicity, and the $[\alpha / \mathrm{Fe}]$ ratio, Trager et al. (2000b) and recently Howell (2005) have noted a strong correlation between the $[\alpha / \mathrm{Fe}]$ ratio and the stellar velocity dispersion, but the metallicity $-\sigma_{*}$ correlation appears to be weak. This fact provokes a revision of the formation models for elliptical galaxies. For example, Pipino \& Matteucci (2004) introduce an "inverse wind" or a direct relation between the stellar velocity dispersion and star formation efficiency in every location within a proto-elliptical galaxy. High star-formation efficiency in the densest regions means the shortest epoch of the main star formation, hence the highest $[\alpha / \mathrm{Fe}]$ ratio that is consistent with the observational data; however, the physical base of this relation remains unclear.

We have plotted our results concerning the relation between the metal-line indices and the mean stellar metallicity with the

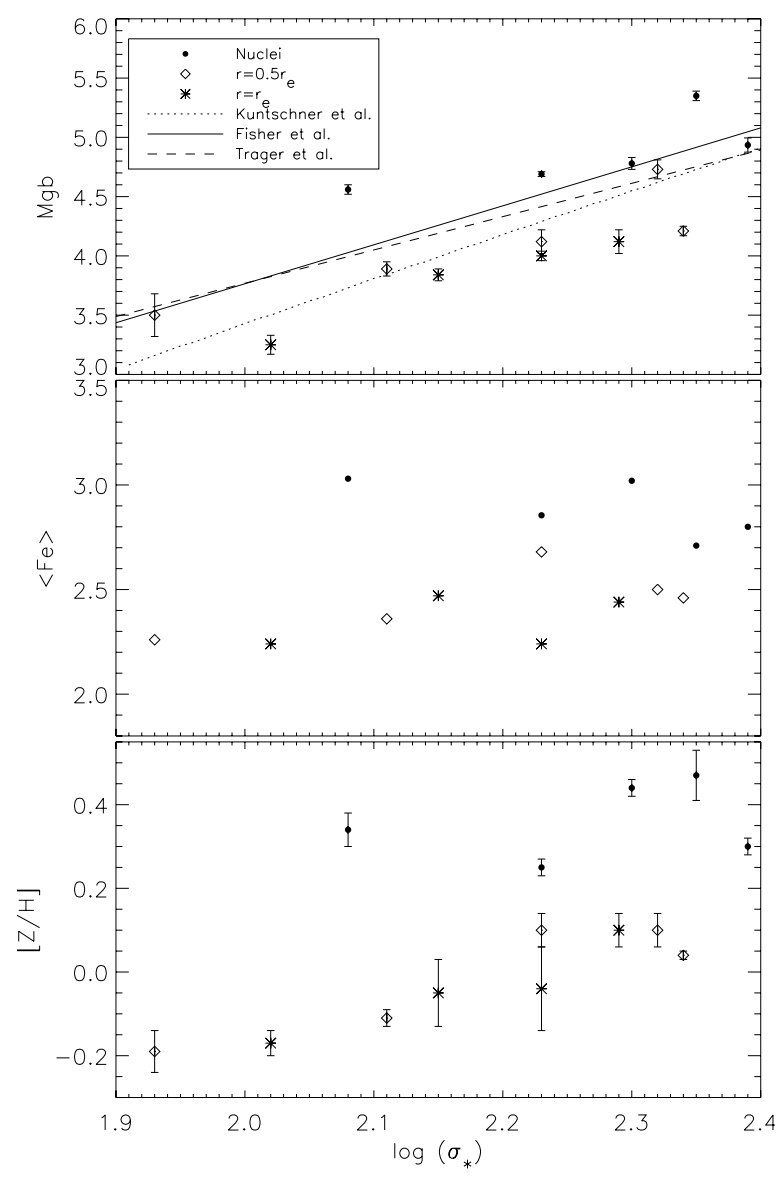

Fig. 12. The correlation between the $\mathrm{Mg} \mathrm{b}$ and $\langle\mathrm{Fe}\rangle$ indices and metallicity and the local stellar velocity dispersion in three positions along the radii in galaxies: in the nuclei, at $r=0.5 r_{\mathrm{e}}$, and at $r=r_{\mathrm{e}}$.

stellar velocity dispersion in Fig. 12; we have plotted not only the nuclei, but also the characteristics at $r=0.5 r_{\mathrm{e}}$ and at $r=r_{\mathrm{e}}$. In the top plot, where we compare the $\mathrm{Mg} b$ index and the stellar velocity dispersion, we also indicate the mean relations found earlier by Fisher et al. (1995), Trager et al. (2000b), and Kuntschner et al. (2001). The high accuracy of our data allows us to separate dependencies for the nuclei and for the off-nuclear regions. They go in parallel, with a significant vertical shift. Meanwhile, the relations published so far go much steeper than our observations and "try" to involve all the points that are in fact physically separated. The clearest physical picture is shown by the bottom plot, $[Z / \mathrm{H}]$ vs. $\log \sigma$. The points for $r=0.5 r_{\mathrm{e}}$ and $r=r_{\mathrm{e}}$ are mixed; they represent good correlation, the rms scatter around the linear regression, $[Z / \mathrm{H}]=-1.54+0.69 \log \sigma$, is only 0.04 dex. In contrast, the points for the nuclei are certainly above this relation, and for the nuclei we do not see any correlation between the (nuclear) metallicity and the central stellar velocity dispersion.

The separated position of the nuclear points in Fig. 12 forces us to suggest that the nuclear star formation in elliptical galaxies is never stopped by galactic wind, but instead proceeds quickly and efficiently toward the full exhaustion of the gas. The duration of star formation over the whole galactic body may be limited by the onset of a galactic wind due to the feedback from hot stars and supernovae and is governed by gravitation depending on the depth of the potential well. In any case, our results imply that all the physical correlations must be searched for only among the integrated characteristics of elliptical galaxies. Their stellar 
nuclei have their own evolution and are not representative of the galaxies as a whole.

\section{Conclusions}

By studying the Lick index gradients in 5 elliptical galaxies with moderate luminosity and located in different environments, we have carefully disentangled age-metallicity effects and measured radial variations of the mean luminosity-weighted stellar ages and metallicities up to $0.5 r_{\mathrm{e}}$ in one galaxy, up to $1.3 r_{\mathrm{e}}$ in two galaxies and up to $2 r_{\mathrm{e}}$ in another two galaxies. We have found that the mean stellar age is constant along the radius only in one galaxy out of the 5 . The other 4 galaxies demonstrate quite different mean stellar age behaviour: the outer parts are older than the centres in 3 cases and younger in one case. Among the former, we suspect the influence of the compact, rather young circumnuclear disks in two galaxies. The metallicity gradients cannot be approximated by a single power law over the full extension of the radius in 4 galaxies out of 5 . The inner metallicity gradients within $0.5 r_{\mathrm{e}}$ are all rather steep, steeper than -0.4 metallicity dex per radius dex, and are inconsistent with the origin of the elliptical galaxies by a major merger. The outer parts may represent mostly the mix of stellar populations provided by minor mergers.

Acknowledgements. During the data analysis we have used the LyonMeudon Extragalactic Database (LEDA) supplied by the LEDA team at the CRAL-Observatoire de Lyon (France) and the NASA/IPAC Extragalactic Database (NED) which is operated by the Jet Propulsion Laboratory, California Institute of Technology, under contract with the National Aeronautics and Space Administration. The research is partly based on observations made with the NASA/ESA Hubble Space Telescope, obtained from the data archive at the Space Telescope Science Institute, which is operated by the Association of Universities for Research in Astronomy, Inc., under NASA contract NAS 5-26555. The spectral study of stellar populations in early-type group and cluster galaxies is supported by the Russian Foundation for Basic Researches (05-02-19805-MFa).

\section{References}

Afanasiev, V. L., \& Moiseev, A. V. 2005, AstL, 31, 194 Barnes, J., 1996, IAUS, 171, 191
Beasley, M. A., Brodie, J. P., Strader J., et al. 2004, AJ, 128, 1623 Bekki, K., \& Shioya, Y., 1999, ApJ, 513, 108

Burkhead, M. S., \& Kalinowski, J. K. 1974, AJ, 79, 835

Caon, N., Capaccioli, M., \& D’Onofrio, M. 1994, A\&AS, 106, 199 Carlberg, R. G. 1984, ApJ, 286, 403

Carollo, C. M., Danziger, I. J., \& Buson, L. 1993, MNRAS, 265, 553

Davies, R. L., Sadler, E. M., \& Peletier, R. F. 1993, MNRAS, 262, 650

de Vaucouleurs, G. 1948, Annales d'Astrophysique, 11, 247

Faber, S. M. 1973, ApJ, 179, 731

Fisher, D., Franx, M., \& Illingworth, G. 1995, ApJ, 448, 119

Forbes, D. A., Sánchez-Blázquez, P., \& Proctor, R. 2005, MNRAS, 361, L6

Garcia, A. M. 1993, A\&AS, 100, 47

Giuricin, G., Marinoni, C., Ceriani, L., \& Pisani, A. 2000, ApJ, 543, 178

Gorgas J., Efstathiou, G., Aragon, \& Salamanca, A. 1990, MNRAS, 245, 217

Henry, R.B.C., \& Worthey, G. 1999, PASP, 111, 919

Howell, J. H. 2005, AJ, 130, 2065

Hubble, E. P. 1926, ApJ, 64, 321

Kobayashi, C. 2004, MNRAS, 347, 740

Kobayashi, C., \& Arimoto, N. 1999, ApJ, 527, 573

Kuntschner, H., Lucey, J. R., Smith, R. J., Hudson, M. J., \& Davies, R. L. 2001, MNRAS, 323, 615

Larson, R. B. 1974, MNRAS, 166, 585

Mehlert, D., Thomas, D., Saglia, R. P., Bender, R., \& Wegner, G. 2003, A\&A, 407, 423

Ogando, R. L. C., Maia, M. A. G., Chiappini, et al. 2005, ApJ, 632, L61

Peletier, R. F., \& Valentijn, E. A. 1989, Ap\&SS, 156, 127

Peletier, R. F., Davies, R. L., Illingworth, G. D., Davis, L. E., \& Cawson, M. 1990, AJ, 100, 1091

Pilyugin, L. S., Vílchez, J. M., \& Contini, T. 2004, A\&A, 425, 849

Pipino, A., \& Matteucci, F. 2004, MNRAS, 347, 968

Sandage, A., \& Visvanathan, N. 1978, ApJ, 223, 707

Sandage, A., \& Bedke, J. 1994, The Carnegie Atlas of Galaxies (Washington: Carnegie Inst.)

Serra, P., \& Trager, S. C. 2006, MNRAS, submitted [astro-ph/0610343]

Thomas, D., Maraston, C., \& Bender, R. 2003, MNRAS, 339, 897

Tifft, W. G. 1969, AJ, 74, 354

Trager, S. C., Worthey, G., Faber, S. M., Burstein, D., \& Gonzalez, J. J. 1998, ApJS, 116, 1

Trager, S. C., Faber, S. M., Worthey, G., \& González, J. J. 2000a, AJ, 119, 1645

Trager, S. C., Faber, S. M., Worthey, G., \& González, J. J. 2000b, AJ, 120, 165

Trujillo, I., Erwin, P., Asensio Ramos, A., \& Graham, A. W. 2004, AJ, 127, 1917

Vader, J. P., Vigroux, L., Lachieze-Rey, M., \& Souviron, J. 1988, A\&A, 203, 217

White, S. D. M. 1980, MNRAS, 191, 1P

Worthey, G. 1994, ApJS, 95, 107

Worthey, G., Faber, S. M., \& Gonzalez, J. J. 1992, ApJ, 398, 69

Worthey, G., Faber, S. M., Gonzalez, J. J., \& Burstein, D. 1994, ApJS, 94, 687 